\title{
INTEGRATED REGISTRATION OF DYNAMIC RENAL PERFUSION MR IMAGES
}

\author{
Ying Sun ${ }^{1}$, Marie-Pierre Jolly ${ }^{2}$, and José M. F. Moura ${ }^{1}$ \\ ${ }^{1}$ Department of Electrical and Computer Engineering, Carnegie Mellon University, PA 15213 \\ ${ }^{2}$ Department of Imaging and Visualization, Siemens Corporate Research, Inc. Princeton, NJ 08540
}

\begin{abstract}
This paper presents an integrated image registration algorithm to correct the motion induced by patient breathing for dynamic renal perfusion MR images. Registration of kidneys through the MR image sequence is a challenging task due to rapidly changing image contrast over the course of contrast enhancement. Our algorithm achieves temporal image registration in a multi-step fashion. We first roughly register the images by detecting large-scale motion, and then refine the registration results by integrating region information and local gradient information with auxiliary image segmentation results. We have tested the proposed algorithm on several real patients and obtained excellent registration results.
\end{abstract}

\section{INTRODUCTION}

Magnetic resonance imaging (MRI) has been used for the assessment of renal perfusion in an accurate and safe manner. In renal perfusion MRI, the abdomen is scanned repeatedly and rapidly following a bolus injection ${ }^{1}$ of a contrast agent. The kinematics of the contrast agent is reflected in the intensity changes of the obtained time series of MR images. Analysis of the dynamic behavior of the signal intensity can provide valuable functional information. Perfusion MR image sequence often suffers from motion induced by breathing during acquisition. To ensure the correspondence of anatomical structures in different time frames, registration of time-series images is necessary. This is a challenging task because the appearance of the kidney changes rapidly over the course of contrast enhancement and therefore it is not possible to use common approach of block matching looking for best match in intensities across frames. In addition, different renal tissue types do not enhance uniformly, which results in a rapidly changing image contrast.

To the best of our knowledge, there has been limited work on the registration of dynamic renal perfusion MR images. An image processing system has been proposed to

\footnotetext{
This work was supported by grants from NIH grant (P41EB001977 and R01EB/AI-00318).

${ }^{1}$ Bolus injection is the injection of a drug (or drugs) in high quantity at once, the opposite of gradual administration.
}

correct organ displacements using model-based segmentation [1]. Recent published work includes a phase difference movement detection method [2] and a semi-automatic contour registration method [3]. These methods all start with a manually drawn kidney contour in one time frame. That initial contour is used to obtain a mask or a model, and then it is propagated to other images in the sequence. In this paper we propose an integrated image registration algorithm that only requires the user to crop a rectangular region of interest (ROI) containing a kidney. In our approach, large-scale motion is identified by maximizing an edge-based consistency metric that is invariant to rapidly changing contrast; the registration results are then refined by integrating region information and edge information with auxiliary image segmentation results. Our experimental results show that by exploiting the invariance of the consistency metric this algorithm can successfully identify the translational motion of the kidney across the image sequence.

The remaining of the paper is organized as follows. In section 2 , we present our integrated image registration algorithm. Section 3 describes the data and presents our experimental results. We conclude the paper in section 4 .

\section{METHOD}

This section describes an integrated image registration algorithm. The most important consideration in the proposed approach is the use of the interdependence between segmentation and registration. Our observation is that registration and segmentation are mutually beneficial. Segmentation helps registration in that informative features in the images can be identified based on segmentation results. These features are required by a large class of image registration methods prior to the registration process. On the other hand, successful registration enables the use of the entire sequence of images in the segmentation.

We use a multi-step approach in which segmentation and registration are interleaved. Figure 1 shows a block diagram of the proposed scheme. The user is only required to crop a ROI containing one kidney in a single high-contrast image. First, we roughly register the selected ROI across the image sequence. Then, we obtain the contours that delineate the boundaries of renal cortex, by segmenting the 


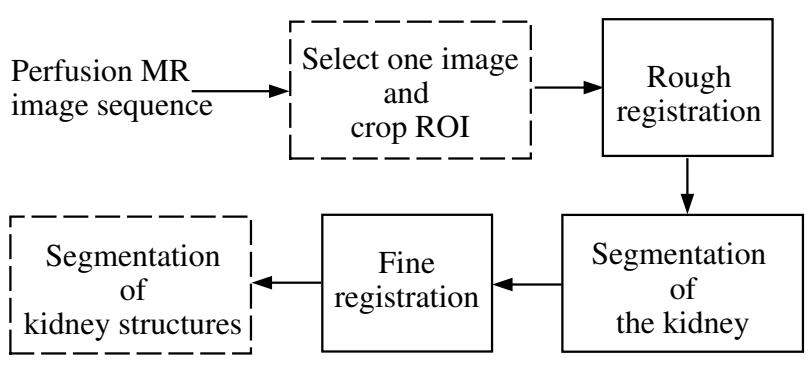

Fig. 1. The block diagram of the proposed algorithm

subtraction image between the high-contrast image and a pre-contrast image. Next, we propagate these contours to other images in the sequence with integer pixel shifts. Finally, the entire image sequence is used to segment different anatomical structures.

\subsection{Rough Registration}

To formulate the registration problem, we start with two assumptions. First, we assume that the kidney is a rigid body, and thus, its shape does not change during the perfusion process. Second, we assume that the motion is only translational while ignoring possible scaling and rotation induced by out-of-plane motion. This is a reasonable assumption because translation is the dominant motion. Given a rectangular ROI in one frame, the goal of this module is to find the best match in other time frames by shifting the bounding box of the selected ROI with integer pixel offsets. In our approach, rough registration is accomplished by template matching.

For template matching, it is important to extract the right features to build the template. Generally two channels of information are used, the intensity and the local gradient. In this case, the intensity changes rapidly and we do not know exactly what intensity level the template should assume. Here, what we really need are image features invariant to rapid intensity and contrast changes. Although the relative intensities between tissues vary with time, we observe that the orientation of the edges along tissue boundaries are always parallel across the image sequence. So we chose the template defined by the image gradient.

In our formulation, the image on which the ROI is manually cropped, is called the reference image. Let $\left(x_{l}^{r}, y_{l}^{r}\right)$ be the position of the $l t h$ pixel inside the ROI. Let $\theta_{l}^{r}$ and $M_{l}^{r}$ stand for respectively the direction and magnitude of the image gradient at the corresponding pixel in the reference image; we obtain $\theta_{l}^{r}$ and $M_{l}^{r}$ using a Sobel edge detector. We have experimentally observed that the final registration results are basically insensitive to the choice of edge detector. Let $\mathcal{S}=\{1,2, \ldots, L\}$ denote the set of indices of all the pixels inside the ROI, our template is represented by a set of four tuples $\left\{\left(x_{l}^{r}, y_{l}^{r}, \theta_{l}^{r}, M_{l}^{r}\right) \mid l \in \mathcal{S}\right\}$. Let $\theta_{c}(x, y)$ denote the edge orientation and $M_{c}(x, y)$ the edge magnitude at pixel $(x, y)$ in the current image; an edge-based consistency metric for each pair of offset $(d x, d y)$ is defined as follows.

$$
C(d x, d y)=\frac{\sum_{l=1}^{L}\left[w_{l}(d x, d y) \cos \left(2 \Delta \theta_{l}(d x, d y)\right)\right]}{\sum_{l=1}^{L} w_{l}(d x, d y)}
$$

with $w_{l}(d x, d y)$ and $\Delta \theta_{l}(d x, d y)$ being the weight and the angle difference, respectively.

$$
\begin{aligned}
w_{l}(d x, d y) & =M_{c}\left(x_{l}^{r}+d x, y_{l}^{r}+d y\right) M_{l}^{r} \\
\Delta \theta_{l}(d x, d y) & =\theta_{c}\left(x_{l}^{r}+d x, y_{l}^{r}+d y\right)-\theta_{l}^{r}
\end{aligned}
$$

This is a weighted sum of $\cos (2 \Delta \theta)$ over the ROI. The cosine of the double angle difference between the current edge orientation and the reference orientation is chosen for two reasons. First, it is invariant to contrast change, i.e., the angle difference between an edge defined by a transition from dark to bright and by a transition from bright to dark is $\Delta \theta=\pi$, which results in $\cos (2 \pi)=\cos (0)$. Second, it is a nonlinear function whose first order derivative peaks at $\pm \pi / 4$, which makes its value relatively less sensitive to disturbances around $\Delta \theta=0, \pi / 2$. Furthermore, we use the product of the edge magnitudes as weight because it is desirable for the ROI to be attracted to strong edges whose orientations are consistent with those of the template. To summarize, using the proposed consistency metric overcomes problems related to the variation in the image contrast.

Since the location of the kidney is confined to a certain range, we only need to compute $C(d x, d y)$ within a searching window. The integer shifts $\left(d x^{*}, d y^{*}\right)$ that maximize $C$ are determined by exploring all possible solutions $(d x, d y)$ over the search space. Although this is an exhaustive search, it is still fast because the search space is limited, usually to $30 \times 10$. Figure 2 displays representative images sampled from a renal perfusion sequence. Results obtained using the proposed method are superimposed by shifting the bounding box to the best match location in each image. As shown, the intensity of the kidney increases as the contrast agent perfuses into the cortex, the medulla, and other structures of the kidney. Despite the rapidly changing contrast and the fact that translational motion of the kidney between two adjacent frames can be considerably large, the algorithm is able to track the kidney reliably in the complete sequence of 250 images, with a maximum tracking error less than 2 pixels in both directions. The tracking error arises in images that lack strong edges because we only use the edge information in the template. To improve the registration accuracy, we propose to integrate the homogeneity of pixel intensities with the edge information by incorporating the knowledge of the contour delineating the kidney boundary.

\subsection{Segmentation of the Kidney}

The purpose of this component is to identify the boundary of the kidney. Since the renal cortex is the outer layer of 


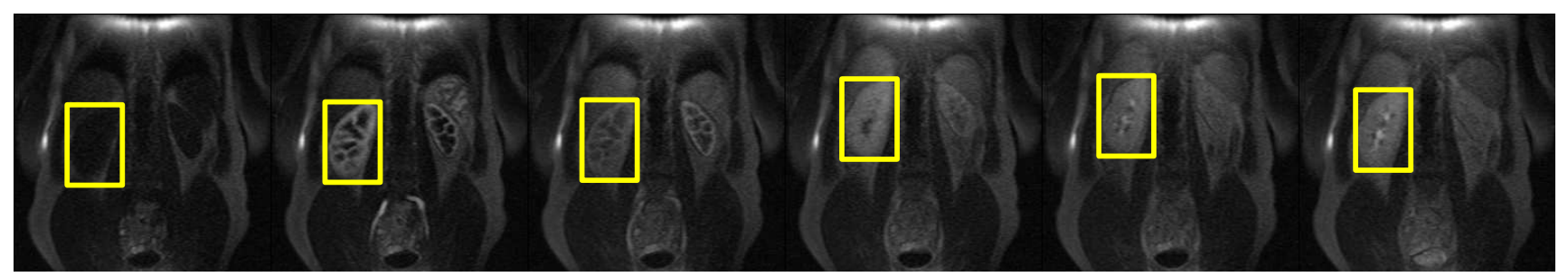

Fig. 2. Rough registration results of representative images selected from a dynamic renal perfusion MR image sequence

the kidney, we attempt to obtain the kidney boundary by segmenting the cortex from the roughly registered image sequence. Figures 3(a)-(c) illustrate three registered ROI representing respectively pre-contrast, wash-in, and postcontrast during a perfusion process. Based on each individual image, it is difficult to distinguish different anatomical structures due to the lack of contrast along some boundary segments. However, subtracting a pre-contrast image, see Figure 3(a), from a high-contrast wash-in image, see Figure 3(b), results in an enhanced image as shown in Figure 3(d). Thanks to the wash-in of the contrast agent, detecting the boundaries of the renal cortex becomes a less challenging problem, to which we can apply, for example, the level set method described in [4]. This is an energy minimization based segmentation method. It assumes that the image is formed by two regions of approximatively piecewise constant intensities of distinct values [4]. In our case, it can be seen easily in Figure 3(d) that the assumption is valid; the image contains a bright object to be detected and a dark background.

The segmentation results obtained using the level set method are overlayed on the subtraction image, see Figure $3(\mathrm{~d})$. Here the dotted contours are the initial curves, while the solid contours are the resulting curves. Although this is an approximate segmentation of the renal cortex, the outer boundary of the kidney is well delineated. Utilization of other images in the sequence, for instance, a post-contrast image shown in Figure 3(c), leads to a more accurate segmentation of the renal cortex. Figure 3(e) shows the final segmentation results by overlaying the contours on an original image from the sequence, while Figure 3(f) displays the mask for renal cortex. These results indicate that we are able to identify the kidney boundary accurately and obtain a mask for the cortex.

\subsection{Fine Registration}

The segmentation results obtained in the previous step make it possible to refine the template by ignoring irrelevant edge information, and at the same time, by incorporating regional homogeneity of pixel intensities.

Recall that the old template described in Section 2.1 is represented by $\left\{\left(x_{l}^{r}, y_{l}^{r}, \theta_{l}^{r}, M_{l}^{r}\right) \mid l \in \mathcal{S}\right\}$. Let $S_{1} \subset \mathcal{S}$ denote the set of indices corresponding to either edge pixels lying on the kidney boundary or their nearest neighbors under a second order neighborhood system. The edge-based consistency metric for the current image is then reduced to

$$
C_{e}(d x, d y)=\frac{\sum_{l \in S_{1}}\left[w_{l}(d x, d y) \cos \left(2 \Delta \theta_{l}(d x, d y)\right)\right]}{\sum_{l \in S_{1}} w_{l}(d x, d y)}
$$

Let $S_{2} \subset \mathcal{S}$ denote the set of $N$ pixels that belong to the cortex mask. Let $m_{c}(d x, d y)=\frac{1}{N} \sum_{l \in S_{2}} I\left(x_{l}^{r}+d x, y_{l}^{r}+d y\right)$ represent the average pixel intensity over the cortex mask in the current image for offset $(d x, d y)$. We define a regionbased consistency metric that penalizes intensity inhomogeneity as:

$$
\begin{aligned}
C_{r}(d x, d y) & =\frac{1-k^{2}(d x, d y)}{1+k^{2}(d x, d y)} \\
k(d x, d y) & =\frac{\sqrt{\frac{1}{N} \sum_{l \in S_{2}}\left[I\left(x_{l}^{r}+d x, y_{l}^{r}+d y\right)-m_{c}(d x, d y)\right]^{2}}}{m_{c}(d x, d y)}
\end{aligned}
$$

Note that $k$ is analogous to the tangent of an angle, which makes $C_{r}$ the cosine of the double angle like $C_{e}$. We combine the above two metrics as a weighted sum to obtain $C^{\prime}(d x, d y)=\lambda_{c} C_{e}(d x, d y)+\left(1-\lambda_{c}\right) C_{r}(d x, d y)$, where $0 \leq \lambda_{c} \leq 1$, and the value of $\lambda_{c}$ is linearly proportional to the average gradient magnitude in the current image. Thus, edge information is dominant in images with strong edges. In contrast, region information plays a more important role in images without strong edges. Similar to section 2.1, the integer shifts can be determined by maximizing $C^{\prime}(d x, d y)$.

Figures 4(a)-(c) compare the results obtained before (thin contours) and after (thick contours) applying the fine registration algorithm. For some images, as in Figure 4(a), the thick contour is identical to the thin contour; For other images, the thick contours delineate the kidney boundary more accurately, as in Figures 4(b)-(c).

\section{RESULTS}

We tested our algorithm on 5 real patient perfusion data sets. Each data set contains 4 slices. The images were acquired on Siemens Sonata MR scanners following bolus injection of Gd-DTPA contrast agent. The image matrix was $256 \times$ 256 pixels. The number of frames in each image sequence ranges from 150 to 350 . 


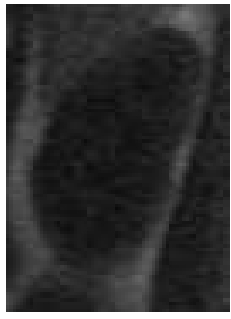

(a)

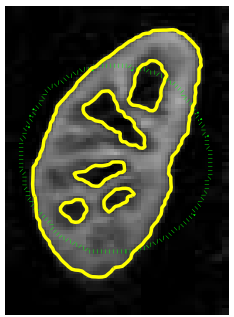

(d)

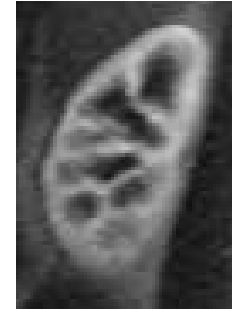

(b)

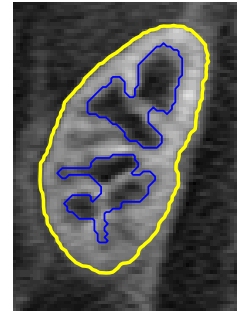

(e)

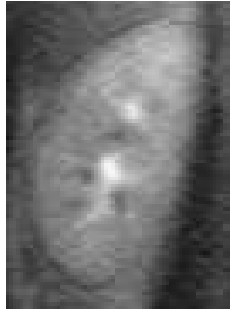

(c)

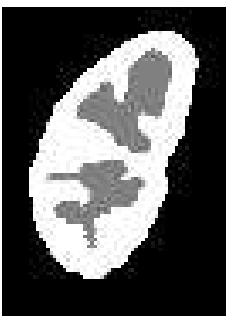

(f)
Fig. 3. Segmentation results of roughly registered images.

To illustrate the performance of the proposed algorithm, we present the results for a real patient in Figures 5(a)(c). The algorithm was performed separately for the left and right kidneys. As shown, it has successfully identified the boundaries (solid contours) of both kidneys in all the images acquired before, during, and after contrast enhancement. The results are more expressive with a video, which is available from www.ece.cmu.edu/ yings/Results.html.

For all the data sets in our study, we observe that the integer pixel shifts obtained using the proposed algorithm are highly consistent with the actual shifts. We have also validated the registration results quantitatively for one sequence of 150 images, by comparing the resulting integer pixel shifts with a "gold standard," i.e., pixel shifts obtained manually. An error size of at most one pixel is obtained for over $95 \%$ of the images.

\section{CONCLUSION}

We have presented an integrated registration algorithm for dynamic renal perfusion MR images. The algorithm integrates region information and edge information with auxiliary image segmentation results. The strength of the algorithm is in the utilization of image features that are invariant to a rapidly changing contrast. We have obtained excellent results with several real patient data sets. The algorithm developed in this paper for tracking translational integer pixel motion of the kidney is the preprocessing step of a subpixel registration method presented in [5]. In [6], we use these registration results to segment different kidney structures based on the distinct dynamics of their intensity-time curve. In our registration framework, the kidney is assumed to be a rigid body. As future work, we plan to take into ac-

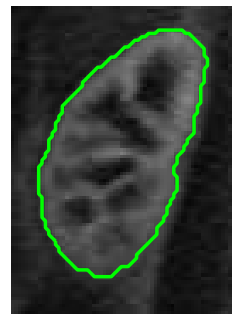

(a)

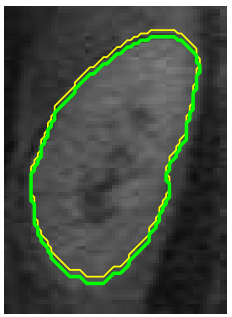

(b)

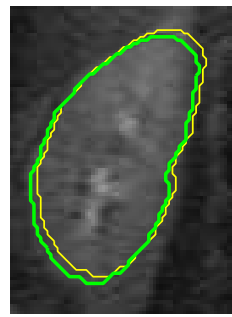

(c)
Fig. 4. Fine registration helps improve the accuracy.

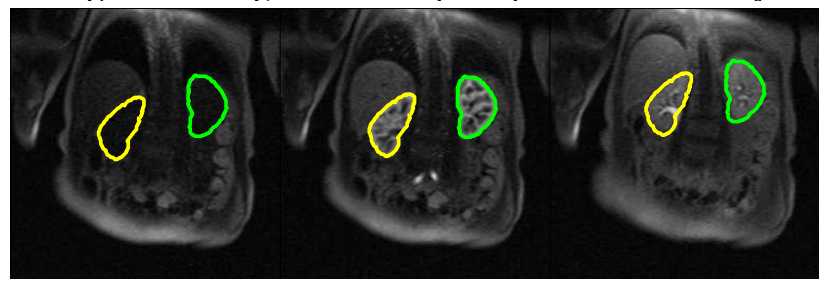

Fig. 5. Results obtained using the proposed integrated registration algorithm on a real patient MR perfusion sequence for both kidneys.

count out-of-plane motion by incorporating parameters for rotation and scaling.

Acknowledgement: We would like to thank Niels Oesingmann from Siemens Medical Systems MR Division, Erlangen, Germany, and Stefan Schoenberg from Institute of Clinical Radiology, Klinikum Grosshadern, Munich, Germany, for providing the data.

\section{REFERENCES}

[1] G. Gerig, R. Kikinis, W. Kuoni, G.K. van Schulthess, and O. Kübler, "Semiautomated ROI analysis in dynamic MRIstudies: Part I: image analysis tools for automatic correction of organ displacements," IEEE Trans. Image Processing, Vol. 11:(2), pp. 221-232, 1992.

[2] E.L.W. Giele, J.A. de Priester, J.A. Blom, J.A. den Boer, J.M.A. van Engelshoven, A. Hasman, and M. Geerlings, "Movement correction of the kidney in dynamic MRI scans using FFT phase difference movement detection," J. Magn Reson Imaging, Vol. 14, pp. 741-49, 2001.

[3] P.J. Yim, H.B. Marcos, M. McAuliffe, D. McGarry, I. Heaton, and P.L. Choyke, "Registration of time-series contrast enhanced magnetic resonance images for renography," in Proc. 14th IEEE Symp. Computer-Based Medical Systems, pp. 516-520, 2001.

[4] T. F. Chan and L. A. Vese, "Active contour without edges," IEEE Trans. Image Processing, Vol.10:(2), pp. 266-77, 2001.

[5] Y. Sun, J. M. F. Moura, and C. Ho, "Subpixel registration in renal perfusion MR image sequence," in Proc. 2004 IEEE Int. Symp. Biomedical Imaging, Arlington, VA, April 2004.

[6] Y. Sun, J. M. F. Moura, D. Yang, Q. Ye, and C. Ho, "Kidney segmentation in MRI sequences using temporal dynamics," in Proc. 2002 IEEE Int. Symp. Biomedical Imaging, Washington, D.C., July 2002. 